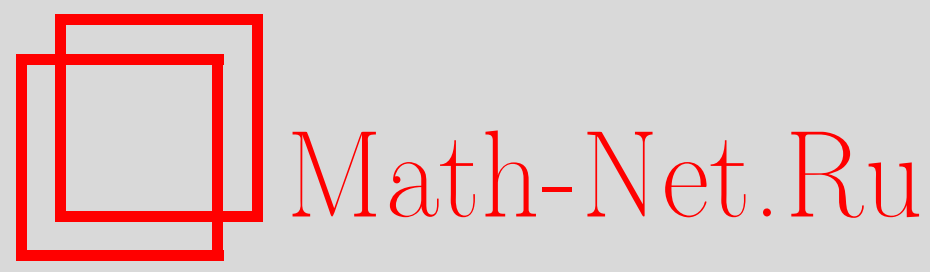

М. И. Рожков, О некоторых классах нелинейных регистров сдвига, обладающих одинаковой цикловой структурой, Дискрет. матем., 2010, том 22, выпуск 2, 96-119

DOI: https://doi.org/10.4213/dm1098

Использование Общероссийского математического портала Math-Net.Ru подразумевает, что вы прочитали и согласны с пользовательским соглашением http://www . mathnet.ru/rus/agreement

Параметры загрузки:

IP: 35.173 .219 .149

26 апреля 2023 г., 13:51:23 


\title{
О некоторых классах нелинейных регистров сдвига, обладающих одинаковой цикловой структурой
}

\author{
() 2010 г. М. И. Рожков
}

\begin{abstract}
Работа посвящена вопросам исследования структуры переходов (цикловой структуры) автономного автомата $R(t)=R\left(G^{n}, \delta_{f}\right)$, называемого регистром сдвига с обратной связью $\left.f, f: G^{n} \rightarrow G\right)$, функция переходов которого $\delta_{f}$ задается следующим образом:

$$
\delta_{f}\left(y_{1}, y_{2}, \ldots, y_{n}\right)=\left(y_{2}, y_{3}, \ldots, y_{n}, f\left(y_{1}, y_{2}, \ldots, y_{n}\right)\right) .
$$

Одной из важных задач в данном направлении исследований является построение нелинейных автоматов $R(f)$, обладающих заданной цикловой структурой, в частности, обладающих циклом длины $2^{n}$ или $2^{n}-1$.
\end{abstract}

\section{1. Введение}

Наиболее универсальный из известных к настоящему моменту методов построения нелинейных двоичных регистров сдвига с заданной цикловой структурой основан на принципе склейки-расклейки циклов исходного регистра сдвига с известной цикловой структурой [7]. При этом эффект склейки двух циклов в один или расклейки одного цикла на два достигается путем инвертирования значения функции обратной связи $g$ исходного автомата $R(g)$ на векторах вида $(1 \alpha)$ и $(0 \alpha)$, где $\alpha \in\left(F_{2}\right)^{n-1}$.

Недостатком данного подхода является то, что, если число этапов склейки-расклейки мало по сравнению с числом $2^{n}$ состояний рассматриваемых автоматов, то существенные части циклов исходного и результирующего автоматов будут совпадать. Другими словами, результирующий автомат будет мало отличаться от исходного. В противном же случае фактически будет иметь место табличное задание функции обратной связи результирующего регистра сдвига с необходимостью использования памяти объемом $2^{n}$ бит.

В работе [11] предложен метод построения полноцикловых регистров $R(f)$ длины $m$ из полноцикловых регистров длины $m-1$. Этот метод, однако, не приводит к увеличению числа полноцикловых регистров длины $m$ по сравнению с исходным числом полноцикловых регистров длины $m-1$.

Известны также методы построения полноцикловых регистров на основе достаточно сложных итеративных процедур, которые не приводят к явной аналитической структуре функции обратной связи построенного регистра сдвига (см. [6]).

Изучению цикловой структуры нелинейных регистров сдвига посвящены также работы [8-10, 12-14] и ряд других. 
В настоящей работе исследуются некоторые классы функций обратной связи $M(f)$, при которых соответствующие регистры сдвига обладают одинаковой цикловой структурой (изоморфны заданному регистру сдвига $R(f)$ ). В этом случае для расчета цикловой структуры всего класса регистров достаточно исследовать структуру одного (наиболее удобного для изучения) представителя. К примеру, все двоичные регистры сдвига длины $n$, обладающие циклом длины $2^{n}-1$, изоморфны друг другу. При этом, как известно, в данном классе регистров сдвига содержатся и линейные автоматы (с помощью которых вырабатываются линейные рекуррентные последовательности максимального периода).

Вместе с тем, описание регистров сдвига, изоморфных заданному регистру $R(f)$, связано со сложной задачей построения специального вида биективных отображений решений уравнений Коши, соответствующих парам изоморфных регистров сдвига.

В настоящей работе получено описание лишь частных классов такого сорта биекций, приведших к построению соответствующих классов изоморфных нелинейных автоматов. При этом наиболее мощные из описанных классов имеют мощность 4(n-2) и отвечают регистрам сдвига с квадратичными функциями обратной связи. Путем исследования на ЭВМ представителей этих классов для $n \leqslant 27$ выделены классы, отвечающие регистрам, которые обладают циклом длины $2^{n}-1$.

\section{2. Условия биективности отображений специального вида}

Будем использовать следующие обозначения: пусть $G^{n}$ - декартово произведение $n$ экземпляров множества $G ; R(f)=R\left(G^{n}, \delta_{f}\right)$ - автономный автомат (регистр сдвига с обратной связью $\left.f: G^{n} \rightarrow G\right)$, функция переходов которого $\delta_{f}$ задается следующим образом:

$$
\begin{array}{rlrl}
\delta_{f}: G^{n} & \rightarrow G^{n}, & \delta_{f}\left(y_{1}, y_{2}, \ldots, y_{n}\right) & =\left(y_{2}, y_{3}, \ldots, y_{n}, f\left(y_{1}, y_{2}, \ldots, y_{n}\right)\right), \\
y_{1}, \ldots, y_{n} & \in G ; \\
K[f, A]: G^{n} & \rightarrow G^{n}, & K f, A](x) & =\left(f(x), f(A(x)), \ldots, f\left(A^{n-1}(x)\right)\right), \\
x & \in G^{n}, \\
A: G^{n} & \rightarrow G^{n}, & \\
f: G^{n} & \rightarrow G ; &
\end{array}
$$

$R\left\{i_{1}, i_{2}, \ldots, i_{m}\right\}-$ множество регулярных (подстановочных) автоматов $R(f)$, цикловая структура которых состоит из $i_{j}$ циклов длины $j, j=1,2, \ldots, m ; Q_{A}$ - множество функций $f: G^{n} \rightarrow G$, отвечающих биективным отображениям $K[f, A]$ при фиксированном отображении $A: G^{n} \rightarrow G^{n} ;|M|$ - мощность конечного множества $M$.

Определение 1. Функцию $f: G^{n} \rightarrow G$ будем называть допустимой, если существует биективное отображение $F=\left(F_{1}, F_{2}, \ldots, F_{n}\right): G^{n} \rightarrow G^{n}$, у которого координатная функция $F_{1}$ совпадает с $f$.

Определение 2. Отображения $B_{1}, B_{2}: G^{n} \rightarrow G^{n}$ назовем эквивалентными (изоморфными), если для некоторой биекции $D: G^{n} \rightarrow G^{n}$ выполняется равенство $D B_{1} D^{-1}=B_{2}$.

Лемма 1. Пусть $A=\left(A_{1}, A_{2}, \ldots, A_{n}\right), B=\left(B_{1}, B_{2}, \ldots, B_{n}\right)-$ произвольнье отображения $G^{n} \rightarrow G^{n}, A_{i}, B_{j}: G^{n} \rightarrow G, f: G^{n} \rightarrow G$. Тогда равенство $A B=\delta_{f} A$ выполняется в том и только в том случае, когда

$$
A_{i}(x)=A_{1}\left(B^{i-1}(x)\right), \quad i=1, \ldots, n, \quad f(A(x))=A_{1}\left(B^{n}(x)\right) .
$$


Справедливость леммы легко усматривается при использовании координатного представления соответствующих отображений.

Лемма 1 свидетельствует о том, что задача построения преобразований $\delta_{f}$, цикловая структура которых совпадает с цикловой структурой заданного отображения $B: G^{n} \rightarrow G^{n}$, самым тесным образом связана с построением биективных отображений вида $K[\varphi, B]$.

Лемма 2. Множество всех биекичй $K[f, A]: G^{n} \rightarrow G^{n}$ есть объединение множеств решений уравнений Коши

$$
X A X^{-1}=\delta_{\varphi}
$$

по всем регистрам сдвига с обратной связью $\varphi: G^{n} \rightarrow G$.

Доказательство. Пусть $X=\left(X_{1}, X_{2}, \ldots, X_{n}\right)$, где $X_{i}: G^{n} \rightarrow G$, является решением уравнения (1). Тогда $X=K\left[X_{1}, A\right]$ в силу леммы 1. С другой стороны, если $K[f, A]-$ биекция, то она является решением уравнения (1) при

$$
\varphi(y)=f\left(A^{n} K^{-1}[f, A](y)\right) .
$$

Теорема 1. Пусть ичикловая структура биективного отображения $A: G^{n} \rightarrow G^{n}$ состоит из $i_{j}$ ичиклов длиньл $j, j=1,2, \ldots,|G|^{n}$. Тогда для числа функций $f: G^{n} \rightarrow G$, отвечающих биективным отображениям $K[f, A]$, справедлива формула

$$
\left|Q_{A}\right|=\left|R\left\{i_{1}, i_{2}, \ldots, i_{m}\right\}\right| \prod_{j=1}^{m}\left(i_{j}\right) ! j^{i_{j}}, \quad m=|G|^{n} .
$$

Доказательство. Заметим, что множества решений уравнения (1) при различных $\varphi: G^{n} \rightarrow G$ не пересекаются. Тогда справедливость теоремы вытекает из леммы 2 и известной формулы для числа решений уравнения Коши.

Следствие 1. Пусть $G=F=\{0,1\}$, и выполнено одно из следующих условий:

(1) Подстановка А состоит из одного ичила длины $2^{n}$.

(2) Подстановка А состоит из одного ичикла длины $2^{n}-1$ и ичикла длиньл 1.

Тогда число булевых функиий $f=f\left(x_{1}, x_{2}, \ldots, x_{n}\right)$, отвечающих биективным отображениям $K[f, A]$, в первом случае равно $2^{2^{n-1}}$, а во втором случае равно $2 \cdot 2^{2^{n-1}}\left(1-2^{-n}\right)$.

Доказательство. Справедливость данного следствия вытекает из формулы (2) с учетом того, что число полноцикловых двоичных регистров сдвига длины $n$ равно $2^{-n} 2^{2^{n-1}}$ (см. [3]), а число регистров, обладающих циклом длины $2^{n}-1$, ровно в два раза больше (каждый такой регистр получается из полноциклового регистра изменением значения его функции обратной связи $f=f(x)=f\left(x_{1}, x_{2}, \ldots, x_{n}\right)$ на одном из векторов $x=(0,0, \ldots, 0)$ и $x=(1,1, \ldots, 1))$.

Следствие 2. Пусть $G=F_{2}=\{0,1\}, n=p-$ простое число, $A=T:\left(F_{2}\right)^{n} \rightarrow\left(F_{2}\right)^{n}-$ преобразование ичиклического сдвига координат векторов. Тогда число булевых функций $f=f\left(x_{1}, \ldots, x_{n}\right)$, отвечающих биективным отображениям $K[f, T]$, равно

$$
\left|Q_{T}\right|=2 p^{\alpha} \alpha !, \quad \alpha=\left(2^{p}-2\right) p^{-1} .
$$


Доказательство. В силу простоты числа $p$ цикловая структура отображения $T$ состоит из двух циклов длины 1 и $\left(2^{p}-2\right) p^{-1}$ циклов длины $p$. Нетрудно убедиться также, что существует единственный регистр сдвига (а именно, регистр с обратной связью $\left.f\left(x_{1}, x_{2}, \ldots, x_{n}\right)=x_{1}\right)$, цикловая структура которого совпадает с цикловой структурой отображения $T$. Теперь результат следствия вытекает непосредственно из формулы (2).

Следующая теорема утверждает, что множество биекций вида $K[f, A]: G^{n} \rightarrow G^{n}$ при всевозможных функциях $f: G^{n} \rightarrow G$ и биекциях $A: G^{n} \rightarrow G^{n}$ совпадает с множеством вообще всех биективных отображений множества $G^{n}$ на себя.

Теорема 2. Пусть $D=\left(f_{1}, f_{2}, \ldots, f_{n}\right)-$ произвольная биекция $G^{n} \rightarrow G^{n}$. Тогда существует биекция $A: G^{n} \rightarrow G^{n}$ такая, что $D=K\left[f_{1}, A\right]$.

Доказательство. Пусть $\delta_{f}$ - преобразование произвольного регулярного регистра сдвига с обратной связью $f$ (регулярные регистры сдвига, очевидно, существуют; например, с обратной связью $\left.f\left(x_{1}, x_{2}, \ldots, x_{n}\right)=x_{1}\right)$.

Через $A$ обозначим отображение $D^{-1} \delta_{f} D$. Тогда $D A D^{-1}=\delta_{f}$. В таком случае в силу леммы 1 справедливы равенства

$$
f_{i}(x)=f_{1}\left(A^{i-1}(x)\right), \quad i=1,2, \ldots, n,
$$

которые и доказывают совпадение биекций $D$ и $K\left[f_{1}, A\right]$.

Замечание 1. Из доказательства теоремы 2 видно, что множество биекций $A$, при которых справедливо равенство $D=K\left(f_{1}, A\right)$, равномощно множеству регулярных регистров сдвига с одной обратной связью.

\section{3. Композиции функций и их свойства}

Далее полагаем, что множество $G$ является коммутативным кольцом с операциями сложения + и умножения ·. При этом знак умножения, где это не вызывает недоразумений, будем опускать.

Определение 3. Функции $f, \varphi: G^{n} \rightarrow G$ будем называть эквивалентными и использовать обозначение $f \sim \varphi$, если отображения $\delta_{f}$ и $\delta_{\varphi}$ обладают изоморфными структурами переходов, то есть когда для некоторой биекции $X: G^{n} \rightarrow G^{n}$ выполняется уравнение Коши

$$
X \delta_{f} X^{-1}=\delta_{\varphi}
$$

Пусть

$$
\lambda(z)=\sum_{i=1}^{m} a_{i} z^{i-1} \in G[z]
$$

- многочлен над кольцом $G, \varphi: G^{n-m+1} \rightarrow G-$ функция $(n-m+1)$ аргументов, $m \leqslant n$.

Через $\lambda * \varphi$ и $\varphi * \lambda$ будем обозначать отображения (функции) $G^{n} \rightarrow G$ вида

$$
\begin{aligned}
& (\lambda * \varphi)(x)=\sum_{i=1}^{n} a_{i} \varphi\left(x_{i}, x_{i+1}, \ldots, x_{i+n-m}\right), \\
& (\varphi * \lambda)(x)=\varphi\left(A_{1}(x), A_{2}(x), \ldots, A_{n-m+1}(x)\right),
\end{aligned}
$$


где

$$
x=\left(x_{1}, x_{2}, \ldots, x_{n}\right) \in G^{n}, \quad A_{j}(x)=\sum_{i=1}^{m} a_{i} x_{i+j-1}, \quad j=1,2, \ldots, n-m+1 .
$$

Пусть $L: G^{n} \rightarrow G-$ фиксированное отображение,

$$
\lambda(z)=\sum_{i=1}^{m} a_{i} z^{i-1} \in G[z], \quad \varphi: G^{n-m+1} \rightarrow G .
$$

Функцию $f: G^{n} \rightarrow G$ будем называть $(\varphi, \lambda, L)$-композицией или $(\lambda, \varphi, L)$-композицией, если

$$
f(x)=(\varphi * \lambda)(x)+L(x) \quad \text { или } \quad f(x)=(\lambda * \varphi)(x)+L(x),
$$

соответственно. Через $M_{n}\left(t_{1}, t_{2}, \ldots, t_{k}\right), t_{i} \in G$, будем обозначать циклическую $n \times n$ матрицу с первой строкой $\left(t_{1}, t_{2}, \ldots, t_{k}, 0, \ldots, 0\right)$.

Лемма 3. Пусть

$$
\lambda(z)=\sum_{i=1}^{m} a_{i} z^{i-1}, \quad \theta(z)=\sum_{i=1}^{s} \gamma_{i} z^{i-1} \in G[z], \quad \psi: G^{n-m-s+2} \rightarrow G .
$$

Тогда справедливы равенства

(1) $\psi *(\theta \lambda)=(\psi * \theta) * \lambda=(\psi * \lambda) * \theta$;

(2) $(\theta \lambda) * \psi=\lambda *(\theta * \psi)=\theta *(\lambda * \psi)$;

(3) $\lambda *(\psi * \theta)=(\lambda * \psi) * \theta$.

Доказательство. Пусть

$$
\theta(z) \lambda(z)=\sum_{i=1}^{m+s-1} r_{i} z^{i-1}, \quad A\left(y_{1}, \ldots, y_{m}\right)=\sum_{i=1}^{m} a_{i} y_{i} .
$$

Тогда

$$
\begin{aligned}
(A * \theta)\left(x_{1}, \ldots, x_{m+s-1}\right) & =\sum_{i=1}^{m} a_{i} \sum_{j=1}^{s} \gamma_{j} x_{j+i-1} \\
& =\sum_{k=1}^{m+s-1} x_{k} \sum_{i=1}^{k} a_{i} \gamma_{k-i+1}=\sum_{i=1}^{m+s-1} r_{i} x_{s} .
\end{aligned}
$$

С учетом коммутативности кольца $G[z]$ отсюда и вытекают равенства п. 1 .

Аналогичным образом получаем, что

$$
\begin{aligned}
\sum_{i=1}^{m} a_{i} & \left(\sum_{j=1}^{s} \gamma \psi\left(x_{i+j-1}, x_{i+j}, \ldots, x_{i+j+n-m-s}\right)\right) \\
& =\sum_{k=1}^{m+s-1} \psi\left(x_{k}, x_{k+1}, \ldots, x_{k+n-m-s+1}\right)\left(\sum_{i=1}^{k} a_{i} \gamma_{k-i+1}\right) \\
& =\sum_{k=1}^{m+s-1} r_{k} \psi\left(x_{k}, x_{k+1}, \ldots, x_{k+n-m-s+1}\right),
\end{aligned}
$$


что равносильно равенству

$$
\lambda *(\theta * \psi)=(\lambda \theta) * \psi
$$

которое свидетельствует о справедливости равенств п. 2. Точно таким же образом доказывается и равенство п. 3. Отметим, что равенство п. 3 вытекает также из предложения 17 B [15].

Каждой функции $f: G^{n} \rightarrow G$, являющейся $(\varphi, \lambda, L)$-композицией вида (3), поставим в соответствие множество

$$
M_{\lambda}(f)=\bigcup_{\{\theta, \nu ; \theta \nu=\lambda\}}\left\{g_{\theta, \nu}\right\},
$$

состоящее из функций $g_{\theta, \nu}: G^{n} \rightarrow G$ вида

$$
g_{\theta, v}=\theta *(\varphi * v)+L,
$$

где многочлены $\theta, v$ и $\lambda$ удовлетворяют равенству $\theta v=\lambda$.

Определение 4. Отображение $\sigma: G \rightarrow G$ назовем аддитивным, если при любых $x, y \in G$ выполняется равенство

$$
\sigma(x+y)=\sigma(x)+\sigma(y) .
$$

Теорема 3. Пусть $\beta_{i}, i=1,2, \ldots, m$, - аддитивные, $a \alpha_{j}, j=1,2, \ldots, n-$ произвольные отображения множества $G$ в себя, $m \leqslant n$, причем любое $\beta_{i}$ перестановочно с каждым из $\alpha_{j}$. Пусть при этом для функиий $h, g: G^{n} \rightarrow G$ имеют место представления

$$
\begin{aligned}
& h(x)=\sum_{i=1}^{m} \beta_{i}\left(\varphi\left(x_{i}, x_{i+1}, \ldots, x_{i+n-m}\right)\right)+\sum_{i=1}^{n} \alpha_{i}\left(x_{i}\right), \\
& g(x)=\varphi\left(\sum_{i=1}^{m} \beta_{i}\left(x_{i}\right), \sum_{i=1}^{m} \beta_{i}\left(x_{i+1}\right), \ldots, \sum_{i=1}^{m} \beta_{i}\left(x_{i+n-m}\right)\right)+\sum_{i=1}^{n} \alpha_{i}\left(x_{i}\right) .
\end{aligned}
$$

Тогда справедливо равенство

$$
A \delta_{h}=\delta_{g} A,
$$

где

$$
\begin{gathered}
A(x)=\left(A_{1}(x), A_{2}(x), \ldots, A_{n}(x)\right), \\
A_{1}(x)=\sum_{i=1}^{m} \beta_{i}\left(x_{i}\right), \quad A_{j+1}(x)=A_{j}\left(\delta_{h}(x)\right), \quad j=1,2, \ldots, n-1 .
\end{gathered}
$$

Доказательство. В силу леммы 1 для доказательства соотношения (5) достаточно убедиться в справедливости равенства

$$
g(A(x))=A_{1}\left(\left(\delta_{h}\right)^{n}(x)\right),
$$

которое равносильно равенству

$$
g(A(x))=\sum_{i=1}^{m} \beta_{i}\left(h\left(\left(\delta_{h}\right)^{i-1}(x)\right)\right) .
$$


Тогда в условиях теоремы правая часть равенства (6) допускает представление

$$
\begin{aligned}
& \sum_{i=1}^{m} \beta_{i}\left(h\left(\left(\delta_{h}\right)^{i-1}(x)\right)\right) \\
& \quad=\sum_{i=1}^{m} \beta_{i}\left[\varphi\left(A_{1}\left(\left(\delta_{h}\right)^{i-1}(x)\right), \ldots, A_{n-m+1}\left(\left(\delta_{h}\right)^{i-1}(x)\right)\right)+\sum_{s=1}^{n} \alpha_{s}\left(x_{s+i-1}\right)\right]
\end{aligned}
$$

где под $x_{n+j}, j=1,2, \ldots, m$, понимаем функцию $h\left(\left(\delta_{h}\right)^{j-1}(x)\right)$. Учитывая перестановочность отображений $\beta_{i}$ и $\alpha_{j}$, а также равенство $A_{i+1}(x)=A_{i}\left(\delta_{h}(x)\right)$, правую часть равенства (6) представляем в виде

$$
\begin{aligned}
\sum_{i=1}^{m} \beta_{i}\left(\varphi \left(A_{i}(x), A_{i+1}(x)\right.\right. & \left.\left.\ldots, A_{i+n-m}(x)\right)\right)+\sum_{s=1}^{n} \alpha_{s}\left(\sum_{j=1}^{m} \beta_{j}\left(x_{s+j-1}\right)\right) \\
& =\sum_{i=1}^{m} \beta_{i}\left(\varphi\left(A_{i}(x), A_{i+1}(x), \ldots, A_{i+n-m}(x)\right)\right)+\sum_{s=1}^{n} \alpha_{s}\left(A_{s}(x)\right),
\end{aligned}
$$

при этом мы использовали соотношение

$$
\sum_{j=1}^{m} \beta_{j}\left(x_{s+j-1}\right)=A_{s}(x)=A_{1}\left(\left(\delta_{h}\right)^{s-1}(x)\right) .
$$

Таким образом, равенство (6) равносильно равенству

$$
g(A(x))=\sum_{i=1}^{m} \beta_{i}\left(\varphi\left(A_{i}(x), A_{i+1}(x), \ldots, A_{i+n-m}(x)\right)\right)+\sum_{s=1}^{n} \alpha_{s}\left(A_{s}(x)\right),
$$

которое очевидным образом выполняется. На этом доказательство завершено.

Замечание 2. Если все $\alpha_{s}, s=1,2, \ldots, n$ являются отображениями в нуль, то теорема 3 будет верна при произвольных (а не только аддитивных) отображениях $\beta_{j}, j=1,2, \ldots, m$.

Замечание 3. Теорема 3 останется, очевидно, верной и для случая произвольной абелевой группы $G$.

Следующие два утверждения содержат условия биективности отображения $A$, фигурирующего в равенстве (5).

Лемма 4. Пусть в условиях теоремы 3 выполнено одно из следующих условий:

(1) $\alpha_{i}=0, i=1,2, \ldots, n, \beta_{1}$ биективно;

(2) $\alpha_{j}=0, j=2,3, \ldots, n-m+1, \beta_{i}=-\beta_{m} \alpha_{n-m+i+1}, i=1,2, \ldots, m-1 ; \alpha_{1} u \beta_{m}$ биективны.

Тогда фигурирующее в равенстве (5) отображение $A: G^{n} \rightarrow G^{n}$ является биективным. 
Доказательство. Пусть выполнено условие 1. Распишем подробнее координатное представление отображения $A(x)=\left(A_{1}(x), A_{2}(x), \ldots, A_{n}(x)\right)$ :

$$
\begin{aligned}
A_{1}(x)= & \sum_{i=1}^{m} \beta_{i}\left(x_{i}\right), \ldots, A_{n-m+1}(x)=\sum_{i=1}^{m} \beta_{i}\left(x_{i+n-m}\right), \\
& \ldots \\
A_{n-m+2}(x)= & \sum_{i=1}^{m-1} \beta_{i}\left(x_{i+n-m+1}\right)+\beta_{m}\left(\varphi\left(A_{1}(x), A_{2}(x), \ldots, A_{n-m+1}(x)\right)\right), \\
A_{n-m+3}(x)= & \sum_{i=1}^{m-2} \beta_{i}\left(x_{i+n-m+2}\right)+\beta_{m-1}\left(\left(\varphi\left(A_{1}(x), A_{2}(x), \ldots, A_{n-m+1}(x)\right)\right)\right. \\
& +\ldots \\
& \ldots \beta_{m}\left(\left(\varphi\left(A_{2}(x), A_{3}(x), \ldots, A_{n-m+2}(x)\right)\right),\right. \\
A_{n}(x)= & \beta_{1}\left(x_{n}\right)+\sum_{i=2}^{m} \beta_{i}\left(\varphi\left(A_{i-1}(x), A_{i}(x), \ldots, A_{i+n-m-1}(x)\right)\right) .
\end{aligned}
$$

Учитывая биективность $\beta_{1}$ по заданным $A_{1}, A_{2}, \ldots, A_{n}$, из последнего равенства возможно однозначное нахождение переменной $x_{n}$.

Аналогичным образом при уже найденных $x_{n-i+1}, x_{n-i+2}, \ldots, x_{n}$ переменная $x_{n-i}$ однозначно находится из $(n-i)$-го уравнения. Таким образом, биективность отображения $A$ для данного случая установлена.

Доказательство второго случая проводится по той же схеме. При этом переменные $x_{1}, x_{2}, \ldots, x_{m-1}$ будут восстановлены соответственно из $(n-m+2)$-го, $(n-m+3)$-го, $\ldots, n$-го уравнений. После чего переменная $x_{m}$ будет найдена из первого уравнения, переменная $x_{m+1}$ из второго и т.д.

Точно таким же образом убеждаемся в справедливости следующего утверждения.

Лемма 5. Пусть в условиях теоремы 3 выполнень условия

(1) $\alpha_{i}=0, i=2,3, \ldots, n и \alpha_{1}(y)=y$ для любого $y \in G$;

(2) биективно аддитивное отображение $\Lambda: G^{n} \rightarrow G^{n}$, задаваемое равенствами

$$
\begin{gathered}
\Lambda(x)=\left(\Lambda_{1}(x), \Lambda_{2}(x), \ldots, \Lambda_{n}(x)\right), \\
\Lambda_{1}(x)=\sum_{i=2}^{m} \beta_{i}\left(x_{i}\right), \quad \Lambda_{j+1}(x)=\Lambda_{j}(T(x)), \quad j=1,2, \ldots, n-1,
\end{gathered}
$$

где $T: G^{n} \rightarrow G^{n}-$ преобразование ичиклического сдвига (влево) координат векторов. Тогда фигурирующее в равенстве (5) отображение $A: G^{n} \rightarrow G^{n}$ является биективным.

Из леммы 5 непосредственно вытекает следующее утверждение.

Следствие 3. Пусть $G$ - коммутативное кольцо с единицей, и в условиях теоремь 3 выполнень условия

(1) $\alpha_{i}=0, i=2,3, \ldots, u \alpha_{1}(y)=$ у для любого $y \in G$; 
(2) $\beta_{i}(x)=b_{i} x, x, b_{i} \in G, i=1,2, \ldots, m$;

(3) определитель цүиклической матрицьь $M_{n}\left(b_{1}, b_{2}, \ldots, b_{m}\right)$ является обратимым элементом кольияа $G$.

Тогда фигурирующее в равенстве (5) отображение $A: G^{n} \rightarrow G^{n}$ является биективным.

Следующая теорема является основным результатом данного раздела.

Теорема 4. Пусть функияия $f: G^{n} \rightarrow G^{n}$ является $(\varphi, \lambda, L)$-композищчией и задана представлением (3). Пусть при этом выполнено одно из условий:

(1) $L=L\left(x_{1}, x_{2}, \ldots, x_{n}\right) \equiv 0, и$ для любого $b \in G$ уравнение $a_{1} y=b$ имеет в $G$ единственное решение,

(2) $L\left(x_{1}, x_{2}, \ldots, x_{n}\right)=x_{1}$, и биективно линейное отображение $G^{n} \rightarrow G^{n}$, соответствует циклической матрице $M_{n}\left(a_{1}, a_{2}, \ldots, a_{m}\right)$.

Тогда все функции множества $M_{\lambda}(f)$ попарно эквивалентны.

Для доказательства нам понадобятся две вспомогательные очевидные леммы.

Лемма 6. Пусть $a=a_{1} a_{2}, a_{1}, a_{2} \in G$ и для любого $b \in G$ уравнение $a x=b$ имеет 8 кольие $G$ единственное решение. Тогда каждое из уравнений $a_{1} x=b$ и $a_{2} x=b$ имеетв кольие $G$ единственное решение.

Лемма 7. Пусть

$$
\begin{array}{lll}
\lambda(z)=\sum_{i=1}^{m} a_{i} z^{i-1}, & \lambda_{1}(z)=\sum_{i=1}^{k} b_{i} z^{i-1}, & \lambda_{2}(z)=\sum_{i=1}^{t} c_{i} z^{i-1}, \\
\lambda(z)=\lambda_{1}(z) \lambda_{2}(z), & \lambda_{i}(z) \in G[z] .
\end{array}
$$

Тогда

$$
M_{n}\left(a_{1}, a_{2}, \ldots, a_{m}\right)=M_{n}\left(b_{1} b_{2}, \ldots, b_{k}\right) \times M_{n}\left(c_{1}, c_{2}, \ldots, c_{t}\right)
$$

(здесь знаком $\times$ обозначено умножение квадратных матрии).

Доказательство теоремь 4. Пусть

$$
\lambda(z)=\lambda_{1}\left(z \lambda_{2}(z)\right)
$$

- произвольное разложение многочлена $\lambda$ на два множителя,

$$
\lambda_{1}(z)=\sum_{i=1}^{k} b_{i} z^{i-1}, \quad \lambda_{2}(z)=\sum_{i=1}^{t} c_{i} z^{i-1},
$$

и выполнено условие 1 теоремы. Тогда в силу леммы 3 и теоремы 3 будет выполнено соотношение

$$
A \delta_{t}=\delta_{g} A
$$

где

$$
\begin{gathered}
A=\left(A_{1}, A_{2}, \ldots, A_{n}\right): G^{n} \rightarrow G^{n}, \quad A_{1}\left(x_{1}, x_{2}, \ldots, x_{n}\right)=\sum_{i=1}^{t} c_{i} x_{i}, \\
A_{j+1}(x)=A_{j}\left(\delta_{t}(x)\right), \quad j=1,2, \ldots, n-1, \quad g=\left(\lambda_{2} *\left(\varphi * \lambda_{1}\right)\right)+L .
\end{gathered}
$$

Заметим, что $a_{1}=b_{1} c_{1}$. Тогда в силу лемм 4 и 6 отображение $A$ биективно, то есть $f \sim g$, и первый случай теоремы доказан. В случае 2 теоремы, биективность отображения $A$ будет вытекать из лемм 5 и 7. На этом доказательство завершено. 


\section{4. Случай поля $G F(2)$}

Введем обозначения, пусть

$e_{1}, e_{2}, \ldots, e_{n}-$ векторы канонического базиса векторного пространства $\left(F_{2}\right)^{n}$,

$e=\sum_{i=1}^{n} e_{i}-$ вектор с единичными координатами,

$E$ - единичное (тождественное) преобразование соответствующего векторного пространства,

$T$ - преобразование циклического сдвига (влево) координат векторов соответствующего векторного пространства.

Кроме того, символом 0 будем обозначать нулевые элементы как поля $F_{2}$, так и векторного пространства $\left(F_{2}\right)^{n}$. В каждом конкретном случае значение этого символа будет видно из контекста.

Отметим, что фигурирующее в равенстве (5) отображение $A$ в случае $G=F_{2}$ представляется в виде

$$
A=\beta_{1} E+\beta_{2} \delta_{h}+\ldots+\beta_{m}\left(\delta_{h}\right)^{m-1}, \quad \beta_{i} \in\{0,1\} .
$$

Теорема 5. Отображение

$$
A=E+\delta_{f}
$$

биективно в том и только том случае, когда функция $f:\left(F_{2}\right)^{n} \rightarrow F_{2}$ удовлетворяет равенству

$$
f(x)=f(x+e) .
$$

Доказательство. Пусть

$$
\psi\left(x_{1}, x_{2}, \ldots, x_{n}\right)=f\left(x_{1}, x_{2}, \ldots, x_{n}\right)+x_{1} .
$$

Биективность отображения $A$ равносильна выполнению неравенства $A(x+y)+A(x) \neq 0$ при всех $x, y \in\left(F_{2}\right)^{n}, y \neq 0$. Так как $y+T(y) \neq e_{n}$ при любом $y \in\left(F_{2}\right)^{n}$, биективность отображения $A$ равносильна выполнению неравенства $\psi(x)+\psi(x+y) \neq 0$ для всех $x \in\left(F_{2}\right)^{n}$ и тех ненулевых $y \in\left(F_{2}\right)^{n}$, при которых $y=T(y)$. Учитывая, что единственным ненулевым решением уравнения $y=T(y)$ является вектор $y=e$, биективность отображения $A$ равносильна выполнению равенства

$$
\psi(x)+\psi(x+e)=1,
$$

которое и приводит к равенству (8).

Заметим, что любую булеву функцию от $n$ переменных, удовлетворяющую равенству (8), можно представить в виде

$$
f\left(x_{1}, x_{2}, \ldots, x_{n}\right)=\varphi\left(x_{1}+x_{2}, x_{2}+x_{3}, \ldots, x_{n-1}+x_{n}\right),
$$


где $\varphi-$ некоторая булева функция от $n-1$ аргументов, однозначно определяемая видом функции $f$. При этом в силу теоремы 3 будет иметь место соотношение

$$
\left(E+\delta_{f}\right) \delta_{t}=\delta_{g}\left(E+\delta_{f}\right),
$$

где

$$
g(x)=\varphi\left(x_{1}, x_{2}, \ldots, x_{n-1}\right)+\varphi\left(x_{2}, x_{3}, \ldots, x_{n}\right) .
$$

Следует отметить, что в некоторых случаях (например, при задании функции $f$ многочленом Жегалкина или табличным образом) достаточно легко убедиться в справедливости тождества (8). В то же время найти вид функции $\varphi$, фигурирующей в представлении (9), может оказаться затруднительным. В этой связи представляет интерес следующее утверждение.

Теорема 6. Пусть функция $f$ удовлетворяет равенству (8), а функиия $g(x)$ представима в виде

$$
g\left(x_{1}, x_{2}, \ldots, x_{n}\right)=f\left(\sum_{i=1}^{n-1} x_{i} \sum_{i=2}^{n-1} x_{i}, \ldots, x_{n-1}, 0\right)+f\left(\sum_{i=2}^{n-1} x_{i} \sum_{i=3}^{n-1} x_{i}, \ldots, 0, x_{n}\right) .
$$

Тогда справедливо равенство

$$
\left(E+\delta_{f}\right) \delta_{t}=\delta_{g}\left(E+\delta_{f}\right) .
$$

Доказательство. Учитывая лемму 1 для доказательства теоремы достаточно показать, что

$$
g(A(x))=f(x)+f\left(\delta_{f}(x)\right),
$$

где $A=E+\delta_{f}$. Справедливы равенства

$$
\begin{aligned}
g(A(x))= & g\left(x_{1}+x_{2}, x_{2}+x_{3}, \ldots, x_{n-1}+x_{n}, x_{n}+f\left(x_{1}, x_{2}, \ldots, x_{n}\right)\right. \\
= & f\left(x_{1}+x_{n}, x_{2}+x_{n}, \ldots, x_{n-1}+x_{n}, 0\right) \\
& \quad+f\left(x_{2}+x_{n}, x_{3}+x_{n}, \ldots, x_{n-1}+x_{n}, 0, x_{n}+f\left(x_{1}, x_{2}, \ldots, x_{n}\right)\right) \\
= & f\left(x_{1}, x_{2}, \ldots, x_{n-1}, x_{n}\right)+f\left(x_{2}, x_{3}, \ldots, x_{n-1}, x_{n}, f\left(x_{1}, x_{2}, \ldots, x_{n}\right)\right) \\
= & f(x)+f\left(\delta_{f}(x)\right) .
\end{aligned}
$$

На этом доказательство завершено.

Теорема 7. Пусть $n>2$ и функция $f:\left(F_{2}\right)^{n} \rightarrow F_{2}$ допускает представление

$$
f(x)=\varphi\left(x_{1}+x_{2}+x_{3}, x_{2}+x_{3}+x_{4}, \ldots, x_{n-2}+x_{n-1}+x_{n}\right)+L(x),
$$

где $x=\left(x_{1}, x_{2}, \ldots, x_{n}\right), \varphi-$ произвольная булева функщия от $n-2$ аргументов,

$$
L\left(x \in\left\{x_{1}+x_{n-2}+x_{n-1}, x_{1}+x_{n-2}+x_{n}, x_{1}+x_{n-1}+x_{n}\right\}\right)
$$

при $n \equiv 0(\bmod 3)$,

$$
L\left(x \in\left\{x_{1}+x_{n-2}+x_{n-1}, x_{1}, x_{1}+x_{n-1}+x_{n}\right\}\right)
$$

nрu $n \equiv 1(\bmod 3)$,

$$
L\left(x \in\left\{x_{1}, x_{1}+x_{n-2}+x_{n}, x_{1}+x_{n-1}+x_{n}\right\}\right)
$$

nрu $n \equiv 2(\bmod 3)$.

Тогда отображение $A=E+\delta_{f}+\left(\delta_{f}\right)^{2}$ является биективным. 
Для доказательства потребуются две леммы.

Лемма 8. Пусть $n \equiv i(\bmod 3), n>2$. Тогда соотношению

$$
T^{2}(y)+T(y)+y=a_{y} e_{n-1}+b_{y} e_{n}, \quad y \in\left(F_{2}\right)^{n}, \quad a_{y}, b_{y} \in F_{2},
$$

удовлетворяют только три отличных от нулевого вектора $y_{1, i}, y_{2, i}, y_{3, i}$, которые в зависимости от $i \in\{0,1,2\}$ имеют вид

$$
\begin{aligned}
& y_{1,0}=011011 \ldots 011, \quad y_{2,0}=101101 \ldots 101, \quad y_{3,0}=110110 \ldots 110 \text {, } \\
& y_{1,1}=0110110 \ldots 011, \quad y_{2,1}=101101 \ldots 1011, \quad y_{3,1}=110110 \ldots 1101 \text {, } \\
& y_{1,2}=011011 \ldots 01101, \quad y_{2,2}=101101 \ldots 10110, \quad y_{3,2}=110110 \ldots 11011 \text {. }
\end{aligned}
$$

При этом элементы $a_{y}, b_{y}$ приведены ниже в таблице:

\begin{tabular}{c|ccccccccc}
$y$ & $y_{1,0}$ & $y_{2,0}$ & $y_{3,0}$ & $y_{1,1}$ & $y_{2,1}$ & $y_{3,1}$ & $y_{1,2}$ & $y_{2,2}$ & $y_{3,2}$ \\
\hline$a_{y}$ & 0 & 0 & 0 & 0 & 1 & 1 & 1 & 0 & 1 \\
\hline$b_{y}$ & 0 & 0 & 0 & 1 & 0 & 1 & 0 & 1 & 1
\end{tabular}

Доказательство. Пусть $n \equiv 0(\bmod 3)$ и для вектора $d=\left(d_{1}, d_{2}, \ldots, d_{n}\right)$ выполняется равенство

$$
T^{2}(d)+T(d)+d=a e_{n-1}+b e_{n}, a, b \in F_{2} .
$$

Тогда

$$
\begin{aligned}
d_{1}+d_{2}+d_{3} & =0, \\
& \cdots \\
d_{n-2}+d_{n-1}+d_{n} & =0, \\
d_{n-1}+d_{n}+d_{1} & =a, \\
d_{n}+d_{1}+d_{2} & =b .
\end{aligned}
$$

Отсюда

$$
\begin{aligned}
& d_{1}=d_{4}=\ldots=d_{n-2}, \\
& d_{2}=d_{5}=\ldots=d_{n-1}, \\
& d_{3}=d_{6}=\ldots=d_{n} .
\end{aligned}
$$

Таким образом, вектор $d$ определяется своими тремя первыми координатами, среди которых должны быть две единицы.

Два других случая леммы доказываются аналогичным образом.

Лемма 9. Пусть $n \equiv i(\bmod 3), n>2 u y_{1, i}, y_{2, i}, y_{3, i}-$ векторы, определеннье $в$ лемме 8. Пусть также для некоторого $j \in\{1,2,3\}$ при всех $\left(x_{1}, x_{2}, \ldots, x_{n}\right) \in\left(F_{2}\right)^{n}$ функция $\psi=x_{1}+f\left(x_{1}, x_{2}, \ldots, x_{n}\right)$ удовлетворяет соотношениям

$$
\begin{aligned}
\psi\left(x+y_{j, i}\right) & =\psi(x)+1, \\
\psi\left(x+y_{j+1, i}\right) & =\psi(x)+1, \\
\psi\left(x+y_{j+2, i}\right) & =\psi(x),
\end{aligned}
$$

где при $j>3$ под $y_{j, i}$ понимается вектор $y_{t, i}, t=j(\bmod 3)+1$. Тогда отображение

$$
A=E+\delta_{f}+\left(\delta_{f}\right)^{2}
$$

является биективным, если выполнено одно из условий: 
(1) $i=0$;

(2) $i=1, j \in\{2,3\}$;

(3) $i=2, j \in\{1,2\}$.

Доказательство. Несложным координатным анализом можно убедиться в том, что отображение $A=E+\delta_{f}+\left(\delta_{f}\right)^{2}$ является биекцией в том и только том случае, когда при всех $x, y \in\left(F_{2}\right)^{n}, y \neq 0$, выполнено соотношение

$$
\begin{aligned}
(\psi(x)+\psi(x+y)) e_{n-1} & +\left(\psi(x)+\psi(x+y)+\psi\left(\delta_{f}(x)\right)+\psi\left(\delta_{f}(x)\right.\right. \\
+ & \left.\left.\left.T(y)+(\psi(x)+\psi(x+y)) e_{n}\right)\right)\right) e_{n} \neq T^{2}(y)+T(y)+y .
\end{aligned}
$$

С учетом леммы 8, для биективности отображения $A$ достаточно, чтобы неравенство (10) выполнялось для любых $y \in\left\{y_{1, i}, y_{2, i}, y_{3, i}\right\}$.

Пусть имеет место условие 1 . Тогда для $y \in\left\{y_{j, 0}, y_{j+1,0}\right\}$ неравенство (10) выполнено, так как в его левой части коэффициент при векторе $e_{n-1}$ равен единице. Пусть теперь $y=y_{j+2,0}$. Учитывая, что $T\left(y_{j+2,0}\right)=y_{j+1,0}$, получаем, что в левой части соотношения (10) коэффициент при векторе $e_{n}$ равен единице. Значит, (10) будет выполняться и в этом случае. Тем самым, лемма при условии 1 доказана. Доказательство для условий 2 и 3 проводится по такой же схеме.

Доказательство теоремь 7. Приступим теперь к доказательству теоремы 7. Если $n(\bmod 3) \neq 0$ и $L=x_{1}$, то с учетом следствия 3 для биективности отображения $E+\delta_{f}+\left(\delta_{f}\right)^{2}$ достаточно показать невырожденность циклической матрицы $M_{n}(1,1,1)$ над полем $F_{2}$. Согласно [4], невырожденность матрицы $M_{n}(1,1,1)$ равносильна взаимной простоте многочленов $1+z+z^{2}$ и $1+z^{n}$ над полем $F_{2}$. А при $n(\bmod 3) \neq 0$ данные многочлены, как нетрудно видеть, взаимно просты.

В остальных случаях для доказательства теоремы 7 достаточно убедиться в справедливости соответствующих условий леммы 8, что не вызывает затруднений.

В следующей теореме наиболее широко отражены условия эквивалентности $(\lambda, \varphi, L)$ и $(\varphi, \lambda, L)$-композиций.

Пусть

$$
\lambda(z)=\sum_{i=1}^{m} a_{i} z^{i-1} \in F_{2}[z], \quad a_{1}=a_{m}=1, \quad m>1 .
$$

Через $\Lambda_{\lambda}$ обозначим подпространство, порожденное (в множестве линейных булевых функций) функциями $\sum_{i=1}^{m} a_{i} x_{i}, \sum_{i=1}^{m} a_{i} x_{i+1}, \ldots, \sum_{i=1}^{m} a_{i} x_{i+n-m}$, и через $\Lambda_{\lambda}(g)-$ смежный класс по подпространству $\Lambda_{\lambda}$, содержащий заданную линейную функцию $g$. Под $(a, b)$ понимаем наибольший общий делитель соответствующих многочленов или чисел.

Теорема 8. Пусть функиия $f:\left(F_{2}\right)^{n} \rightarrow F_{2}$ является $(\varphi, \lambda, L)$-композицичей и задана представлением (3).

Тогда функичи

$$
f(x)=(\varphi * \lambda)(x)+L(x), \quad h(x)=(\lambda * \varphi)(x)+L(x)
$$

являются эквивалентными, если выполнено одно из условий:

(1) $L \in \Lambda_{\lambda}(g), g=x_{1}+\sum_{i=1}^{m-1} a_{i} x_{n-m+i+1}$; 
(2) $L \in \Lambda_{\lambda}(g), g=x_{1}$ и ичиклическая матрицฺа $M_{n}\left(a_{1}, a_{2}, \ldots, a_{m}\right)$ является невырожденной;

(3) $L \in \Lambda_{\lambda}(g), g=x_{1},(\lambda(z), z+1)=1, \lambda(z) \neq \sum_{i=0}^{n-1} z^{i}, n=p-$ простое число, такое, что 2 является первообразным корнем по модулю $p$;

(4) $L \in \Lambda_{\lambda}(g), g=x_{1},(\lambda(z), z+1), n=2^{d}, d \in\{1,2, \ldots\}$;

(5) $L \in \Lambda_{\lambda}(g), g=x_{1}, \lambda(z)=\sum_{i=1}^{m} z^{i-1},(\lambda(z), z+1)=1,(n, m)=1$;

(6) $\lambda(z)=1+z+z^{2}, L \in \Lambda_{\lambda}(g)$, где

$$
\begin{array}{llll}
g(x) \in\left\{x_{1}+x_{n-2}+x_{n-1}, x_{1}+x_{n-2}+x_{n}, x_{1}+x_{n-1}+x_{n}\right\}, & n \equiv 0 & (\bmod 3), \\
g(x) \in\left\{x_{1}+x_{n-2}+x_{n-1}, x_{1}, x_{1}+x_{n-1}+x_{n}\right\}, & n \equiv 1 & (\bmod 3), \\
g(x) \in\left\{x_{1}, x_{1}+x_{n-2}+x_{n}, x_{1}+x_{n-1}+x_{n}\right\}, & n \equiv 2 & (\bmod 3) .
\end{array}
$$

Доказательство. В условиях 1 и 2 теорема следует из теоремы 3 с учетом соответственно лемм 4 и 5. Пусть выполнено условие 3. Тогда (см. [5]) многочлен $z^{n}+1$ имеет только два неприводимых множителя $z+1$ и $1+z+\ldots+z^{n-1}$. Отсюда следует взаимная простота многочленов $\lambda(z)$ и $z^{n}+1$, и следовательно (см. [4]), невырожденность циклической матрицы $M_{n}\left(a_{1}, a_{2}, \ldots, a_{m}\right)$. Тем самым, мы оказываемся в условиях п. 2 . Аналогичным образом показывается, что при выполнении условий 4 и 5 также будут выполнены и условия п. 2. Наконец, если выполнены условия 6, то справедливость теоремы вытекает из теорем 3 и 7.

Следующая теорема является центральной в данном разделе.

Теорема 9. Пусть функция $f:\left(F_{2}\right)^{n} \rightarrow F_{2}$ является $(\varphi, \lambda, L)$-композицичеи и задана представлением (3), $\lambda(z)=g_{1} \cdots g_{k}$ есть разложение многочлена $\lambda(z)$ в произведение неприводимых множителей $g_{i}(z), i=1,2, \ldots, k$ (многочлень $g_{i}$ не обязательно различны).

Пусть при этом для каждого фиксированного $i \in\{1,2, \ldots, k\}$ функция

$$
f(x)=(\varphi * \lambda)(x)+L(x)=\left(\left(\varphi *\left(\lambda / g_{i}\right)\right) * g_{i}\right)+L(x),
$$

рассматриваемая как $\left(\varphi *\left(\lambda / g_{i}\right), g_{i}, L\right)$-композиция, удовлетворяет одному из условий 1-6 (не обязательно одному и тому же при разных $i$ ) теоремы 8.

Тогда функиии множества $M_{\lambda}(f)$ попарно эквивалентны.

Доказательство. Доказательство проведем индукцией по числу множителей $k$. При $k=1$ справедливость теоремы вытекает из теоремы 8. Пусть теорема верна для $k-1$ и $\theta-$ произвольный делитель многочлена $\lambda(z)$. Если $\theta \neq \lambda$, тогда в разложение $\theta$ входит не более $k-1$ множителей, и функция $(\theta *(\varphi *(\lambda / \theta))(x)+L(x)$ эквивалентна функции $f(x)=((\varphi *(\lambda / \theta) * \theta)(x)+L(x)$ по предположению индукции.

Пусть теперь $\theta=\lambda$. Тогда функция

$$
f=\left(\left(\varphi * g_{k}\right) *\left(\lambda / g_{k}\right)\right)(x)+L(x),
$$

являющаяся $\left(\left(\varphi * g_{k}\right),\left(\lambda / g_{k}\right), L\right)$-композицией, эквивалентна

$$
h=\left(\left(\lambda / g_{k}\right) *\left(\varphi * g_{k}\right)\right)(x)+L(x) .
$$


С другой стороны, функция $h$ является $\left(\left(\left(\lambda / g_{k}\right) * \varphi\right), g_{k}, L\right)$-композицией и эквивалентна функции

$$
\begin{aligned}
\left.\left(g_{k} *\left(\lambda / g_{k}\right) * \varphi\right)\right)(x)+L(x) & =\left(\left(g_{k}\left(\lambda / g_{k}\right)\right) * \varphi\right)(x)+L(x) \\
& =(\lambda * \varphi)(x)+L(x)=(\theta * \varphi)(x)+L(x) .
\end{aligned}
$$

Тем самым индуктивный переход завершен, теорема доказана.

\section{5. Оценки мощности классов эквивалентных функций}

Далее будем рассматривать регулярные (подстановочные) регистры сдвига над полем $F_{2}$ и использовать обозначения предыдущего раздела. Известно [7], что необходимым и достаточным условием регулярности двоичного регистра сдвига $R(f)$ (то есть биективности отображения $\delta_{f}$ ) является линейность функции обратной связи $f$ по первой координате:

$$
f\left(x_{1}, x_{2}, \ldots, x_{n}\right)=x_{1}+\psi\left(x_{2}, \ldots, x_{n}\right) .
$$

Так как в случае линейности функции $\varphi$ функции $(\varphi * \lambda)$ и $(\lambda * \varphi)$ совпадают, при рассмотрении композиции вида (3) и (4) всегда будем предполагать, что функция $\varphi$ нелинейна $(\operatorname{deg}(\varphi)>1)$ и не зависит от первой координаты, а функция $L=L\left(x_{1}, x_{2}, \ldots, x_{n}\right)$ является линейной и зависящей существенным образом от переменной $x_{1}$.

Введем несколько дополнительных обозначений. Пусть

$$
\begin{gathered}
s:\left(F_{2}\right)^{n} \rightarrow\left(F_{2}\right)^{n}, \quad s\left(x_{1}, x_{2}, \ldots, x_{n}\right)=\left(x_{n}, x_{n-1}, \ldots, x_{1}\right) ; \\
Z(f)=\left\{f(x), f(x+e)+1, f_{\nabla}(x), f_{\nabla V}(x+e)+1\right\}, \\
f:\left(F_{2}\right)^{n} \rightarrow F_{2}, \quad f\left(x_{1}, x_{2}, \ldots, x_{n}\right)=x_{1}+\psi\left(x_{2}, x_{3}, \ldots, x_{n}\right), \\
x, e \in\left(F_{2}\right)^{n}, \\
f_{\nabla}(x)=f_{\nabla}\left(x_{1}, x_{2}, \ldots, x_{n}\right)=x_{1}+\psi\left(x_{n}+x_{n-1}+\ldots+x_{2}\right) ;
\end{gathered}
$$

$Z_{+}(f), f:\left(F_{2}\right)^{n} \rightarrow F_{2},-$ множество булевых функций от $n$ переменных, представимых в виде $g+h$ при некоторых $g \in Z(f)$ и

$$
h(x)=\sum_{i=1}^{n} h_{i} x_{i}+h_{0}, \quad h_{j} \in\{0,1\} .
$$

Если функция $f:\left(F_{2}\right)^{n} \rightarrow F_{2}$ является $(\varphi, \lambda, L)$-композицией, то

$$
M(f)=\bigcup_{g \in M_{\lambda}(f)} Z(g) .
$$

При заданном многочлене

$$
\lambda(z)=\sum_{i=1}^{m} a_{i} z^{i-1} \in F_{2}[z], \quad a_{1}=a_{m}=1, \quad m>1 .
$$

через $\lambda^{*}(z)$ обозначим многочлен

$$
\lambda^{*}(z)=\sum_{i=1}^{m} a_{m-i+1} z^{i-1} .
$$


Лемма 10. Функции множества $Z(f)$ являются попарно эквивалентными.

Доказательство. Утверждение данной леммы вытекает из легко проверяемых соотношений

$$
\begin{gathered}
\tau \delta_{f} \tau^{-1}=\delta_{g}, \quad g(x)=f(x+e)+1, \quad \tau:\left(F_{2}\right)^{n} \rightarrow\left(F_{2}\right)^{n}, \quad \tau(x)=x+e ; \\
s \delta_{f} s^{-1}=\left(\delta_{h}\right)^{-1}, \\
h(x)=f_{\nabla}(x) .
\end{gathered}
$$

Теорема 10. Пусть функции $f, h:\left(F_{2}\right)^{n} \rightarrow F_{2}$ являются соответственно $(\varphi, \lambda, L)-u$ $(\lambda, \varphi, L)$-композициями и заданы представлениями

$$
\begin{gathered}
f(x)=(\varphi * \lambda)(x)+L(x), \quad h(x)=(\lambda * \varphi)(x)+L(x), \\
\operatorname{deg}(\varphi)>1, \quad \lambda(z)=\sum_{i=1}^{m} a_{i} z^{i-1} \in F_{2}[z], \quad a_{1}=a_{m}=1, \quad m>1 .
\end{gathered}
$$

Тогда функция $h(x)$ не может лежать в множестве $Z_{+}(f)$.

Доказательство. Пусть

$$
f(x)=h(x)+l(x), \quad \operatorname{deg}(l(x)) \leqslant 1 .
$$

Это равносильно равенству

$$
\varphi\left(\sum_{i=1}^{m} a_{i} x_{i+1}, \ldots, \sum_{i=1}^{m} a_{i} x_{i+n-m}\right)=\sum_{i=1}^{m} a_{i} \varphi\left(x_{i+1}, \ldots, x_{i+n-m}\right)+l(x) .
$$

Очевидно, что без потери общности можно ограничиться случаем, когда функция $\varphi\left(z_{2}, z_{3}, \ldots, z_{n-m+1}\right)$ зависит от переменной $z_{n-m+1}$ нелинейным образом. Рассмотрим разложение функции $\varphi$ по координате $z_{n-m+1}$

$$
\varphi\left(z_{2}, z_{3}, \ldots, z_{n-m+1}\right)=\varphi_{0}\left(z_{2}, z_{3}, \ldots, z_{n-m}\right)+z_{n-m+1} \varphi_{1}\left(z_{2}, z_{3}, \ldots, z_{n-m}\right) .
$$

Тогда соотношение (12) принимает вид

$$
\begin{aligned}
& \varphi_{0}\left(\sum_{i=1}^{m} a_{i} x_{i+1}, \ldots, \sum_{i=1}^{m} a_{i} x_{i+n-m-1}\right) \\
&+\left(\sum_{i=1}^{m} a_{i} x_{i+n-m}\right) \varphi_{1}\left(\sum_{i=1}^{m} a_{i} x_{i+1}, \ldots, \sum_{i=1}^{m} a_{i} x_{i+n-m-1}\right) \\
&=\sum_{i=1}^{m} a_{i} \varphi_{0}\left(x_{i+1}, \ldots, x_{i+n-m-1}\right) \\
& \quad+\sum_{i=1}^{m} a_{i} x_{i+n-m} \varphi_{1}\left(x_{i+1}, \ldots, x_{i+n-m-1}\right)+l(x) .
\end{aligned}
$$

Учитывая равенства $a_{1}=a_{m}=1$, отсюда получаем, что

$$
\varphi_{1}\left(x_{m+1}, x_{m+2}, \ldots, x_{n-1}\right)+\varphi_{1}\left(\sum_{i=1}^{m} a_{i} x_{i+1}, \ldots, \sum_{i=1}^{m} a_{i} x_{i+n-m-1}\right)
$$


есть постоянная. Но тождеству (13) не может удовлетворять ни одна отличная от константы функция $\varphi_{1}$. Полученное противоречие доказывает, что равенство (11) невозможно.

Аналогичными рассуждениями доказывается невозможность равенства

$$
f(x)=h(x+e)+l(x), \quad \operatorname{deg}(l(x)) \leqslant 1 .
$$

Покажем теперь невозможность выполнения равенства

$$
f(x)=h_{\bar{V}}(x+e)+l(x), \quad \operatorname{deg}(l(x)) \leqslant 1 .
$$

Для этого достаточно показать невозможность выполнения равенства

$$
\varphi\left(\sum_{i=1}^{m} a_{i} x_{i+1}, \ldots, \sum_{i=1}^{m} a_{i} x_{i+n-m}\right)=\sum_{i=1}^{m} a_{i} \varphi\left(x_{n-i+1}, x_{n-i}, \ldots, x_{m-i+2}\right)+l(x)
$$

ни при какой функции $l(x), \operatorname{deg}(l(x)) \leqslant 1, \operatorname{deg}(\varphi)>1$.

Рассмотрим разложение $\varphi\left(z_{2}, z_{3}, \ldots, z_{n-m+1}\right)$ по координатам $z_{2}$ и $z_{n-m+1}$ :

$$
\varphi\left(z_{2}, z_{3}, \ldots, z_{n-m+1}\right)=\varphi_{0}+z_{2} \varphi+z_{n-m+1} \varphi_{2}+z_{2} z_{n-m+1} \varphi_{3},
$$

где $\varphi_{0}, \varphi_{1}, \varphi_{2}, \varphi_{3}-$ функции переменных $z_{3}, z_{4}, \ldots, z_{n-m}$. Тогда из (14) получаем, что

$$
\begin{aligned}
\varphi_{3} & =0, \\
\varphi_{1}\left(\sum_{i=1}^{m} a_{i} x_{i+2}, \ldots, \sum_{i=1}^{m} a_{i} x_{i+n-m-1}\right) & =\varphi_{2}\left(x_{n-m}, x_{n-m-1}, \ldots, x_{3}\right)+c_{1}, \\
\varphi_{2}\left(\sum_{i=1}^{m} a_{i} x_{i+2}, \ldots, \sum_{i=1}^{m} a_{i} x_{i+n-m-1}\right) & =\varphi_{1}\left(x_{n-1}, x_{n-2}, \ldots, x_{m+2}\right)+c_{2},
\end{aligned}
$$

где $c_{1}$ и $c_{2}-$ некоторые константы. Пусть равенствам (16) и (17) удовлетворяют функции $\varphi_{1}\left(z_{3}, z_{4}, \ldots, z_{n-m-s}\right), \varphi_{2}\left(z_{t}, z_{t+1}, \ldots, z_{n-m}\right)$, такие, что $\varphi_{1}$ существенно зависит от $z_{n-m-s}$, а $\varphi_{2}$ существенно зависит от $z_{t}, t \geqslant 3$. Тогда в многочлен Жегалкина левой части равенства (16) будут входить члены, содержащие переменную $x_{n-s-1}$. При этом переменных $x_{n-1}, x_{n-2}, \ldots, x_{n-s+1}, x_{n-s}$ в левой части равенства (16) не будет. Аналогичным образом, правая часть равенства (16) будет существенным образом зависеть от переменной $x_{n-m-t+3}$ и не будет зависеть от $x_{n-m-t+4}, x_{n-m-t+5}, \ldots, x_{n-1}$. Таким образом, должно выполняться равенство

$$
n-s-1=n-m-t+3 .
$$

С другой стороны, левая часть равенства (17) будет существенно зависеть от $x_{t}$ и не зависеть от $x_{1}, x_{2}, \ldots, x_{t-1}$, а правая часть данного равенства будет существенно зависеть от $x_{m+s+2}$ и не зависеть от $x_{1}, x_{2}, \ldots, x_{m+s+1}$. Таким образом, должно выполняться также равенство

$$
t=m+s+2 \text {. }
$$

Из (18)-(19) вытекает, что $m=1$. Полученное противоречие показывает, что функции $\varphi_{1}$ и $\varphi_{2}$ являются константами. В таком случае соотношение (14) перейдет в аналогичное соотношение для функции $\varphi_{0}$ от меньшего числа аргументов. Продолжая рассуждения далее, получим, что выполнение равенства (14) возможно лишь при $\operatorname{deg}(\varphi) \leqslant 1$. 
Аналогичными рассуждениями показывается невозможность выполнения равенства

$$
f(x)=h_{\bar{V}}(x+e)+l(x), \quad \operatorname{deg}(l(x)) \leqslant 1 .
$$

На этом доказательство теоремы завершено.

Лемма 11. Пусть функции $f, h:\left(F_{2}\right)^{n} \rightarrow F_{2}$ определены в условиях теоремы 10 и при этом функции $f(x)$ и $f(x+e)+1$ различны. Тогда функиии $h(x) u h(x+e)+1$ также будут различными.

Доказательство. Рассмотрим случай $(\lambda(z), z+1)=1$. Тогда из различности функций $f(x)$ и $f(x+e)+1$ вытекает, что существует вектор $y=\left(y_{2}, y_{3}, \ldots, y_{n-m+1}\right)$, при котором

$$
\varphi(y)+\varphi(y+e)=L(e) .
$$

Если при этом $\varphi(y)+\varphi(y+e)-$ не константа, то

$$
h(x)+h(x+e)=\sum_{i=1}^{m} a_{i} \psi\left(x_{2}, x_{3}, \ldots, x_{n-m+1}\right)+L(e),
$$

где

$$
\psi(z)=\varphi(z)+\varphi(z+e) .
$$

Если предположить, без потери общности, существенную зависимость функции $\psi\left(x_{2}, x_{3}, \ldots, x_{n-m+1}\right)$ от переменной $x_{n-m+1}$, то из (21) получаем существенную зависимость функции $h(x)+h(x+e)$ от переменной $x_{n}$, то есть функция $h(x)+h(x+e)+1$ отлична от константы.

Пусть теперь равенство (20) верно при любых векторах $y$. Учитывая, что условие $(\lambda(z), z+1)=1$ равносильно условию $\sum_{i=1}^{m} a_{i}=1$ (суммирование в поле $F_{2}$ ), получаем, что

$$
h(x)+h(x+e)+1=L(e)\left(\sum_{i=1}^{m} a_{i}\right)+L(e)+1=1 .
$$

Если же $(\lambda(z), z+1)=z+1$, то из условия $f(x) \neq f(x+e)+1$ вытекает равенство $h(0)+h(e)+1=1$, и лемма полностью доказана.

Лемма 12. Пусть в условиях теоремы 10 выполняется равенство $\lambda^{*}(z)=\lambda(z)$. Тогда функции $h(x)$ и $h_{\nabla}(x)$ совпадают в том и только в том случае, когда совпадают функции $f(x) u f_{\nabla}(x)$.

Доказательство. Запишем равенства

$$
f(x)=f_{\nabla}(x), \quad h(x)=h_{\nabla}(x)
$$

в следующей эквивалентной форме:

$$
\begin{aligned}
\varphi\left(\sum_{i=1}^{m} a_{i} x_{i+1}, \ldots, \sum_{i=1}^{m} a_{i} x_{i+n-m}\right) & +\varphi_{\leftrightarrow}\left(\sum_{i=1}^{m} a_{i} x_{i+1}, \ldots, \sum_{i=1}^{m} a_{i} x_{i+n-m}\right) \\
& =L(x)+L_{\nabla}(x), \\
\sum_{i=1}^{m} a_{i}\left(\varphi\left(x_{i+1}, x_{i+2}, \ldots, x_{i+n-m}\right)\right) & \left.+\varphi_{\leftrightarrow}\left(x_{i+1}, x_{i+2}, \ldots, x_{i+n-m}\right)\right) \\
& =L(x)+L_{\nabla}(x),
\end{aligned}
$$


где под $\varphi_{\leftrightarrow}\left(z_{1}, z_{2}, \ldots, z_{n-m}\right)$ понимается функция $\varphi\left(z_{n-m}, z_{n-m-1}, \ldots, z_{1}\right)$.

Рассмотрим далее случай, когда

$$
\varphi\left(z_{1}, z_{2}, \ldots, z_{n-m}\right)+\varphi_{\leftrightarrow}\left(z_{1}, z_{2}, \ldots, z_{n-m}\right)=\sum_{i=1}^{n-m} b_{i} z_{i}, \quad b_{i} \in F_{2} .
$$

Тогда соотношения (22)-(23) являются эквивалентными, поскольку каждое из них эквивалентно соотношению

$$
\sum_{i=1}^{m} a_{i} \sum_{j=1}^{n-m} b_{j} x_{i+j}=L(x)+L_{\nabla}(x) .
$$

Теперь рассмотрим случай, когда (24) не имеет места. Тогда без потери общности можно полагать, что функция $\varphi\left(z_{1}, z_{2}, \ldots, z_{n-m}\right)+\varphi \leftrightarrow\left(z_{1}, z_{2}, \ldots, z_{n-m}\right)$ нелинейным образом зависит от переменной $z_{n-m}$. В таком случае левые части соотношений (22) и (23) будут нелинейным образом зависеть от переменной $x_{n}$. Следовательно, ни одно из равенств (22)-(23) не будет верным. На этом доказательство завершено.

Лемма 13. Пусть в условиях теоремь 10 имеет место одно из дополнительных условий:

(1) $\lambda^{*}(z)=\lambda(z),(\lambda(z), z+1)=z+1, L(e)=0$;

(2) $\lambda^{*}(z)=\lambda(z),(\lambda(z), z+1)=1$.

Тогда в первом случае $f(x) \neq f_{\nabla}(x+e)+1 u h(x) \neq h_{\nabla}(x+e)+1$, а во втором случае $h(x)$ и $f_{\nabla}(x+e)+1$ совпадают в том и только в том случае, когда совпадают функции $f(x) u f_{\nabla}(x+e)+1$.

Справедливость леммы для случая 1 вытекает при сравнении значений соответствующих функций в точке $x=0 \in\left(F_{2}\right)^{n}$. Доказательство для случая 2 проводится по той же схеме, что и леммы 12 , и мы его опускаем.

Следующая теорема является основной в данном разделе.

Теорема 11. Пусть функция $f:\left(F_{2}\right)^{n} \rightarrow F_{2}$ является $(\varphi, \lambda, L)$-композицией и задана представлением

$$
f(x)=(\varphi * \lambda)(x)+L(x),
$$

где

$$
\lambda(z)=\sum_{i=1}^{m} a_{i} z^{i-1} \in F_{2}[z], \quad a_{1}=a_{m}=1, \quad m>1,
$$

функиия $\varphi=\varphi\left(z_{1}, z_{2}, \ldots, z_{n-m+1}\right)$ нелинейна, $\operatorname{deg}(\varphi)>1$, и не зависит от первой координаты, а функиия $L=L\left(x_{1}, x_{2}, \ldots, x_{n}\right)$ является линейной и зависящей существенным образом от переменной $x_{1}$.

Пусть при этом выполнены дополнительные условия:

(1) $\lambda(z)=\theta^{t}(z), t-$ целое положительное число, $\theta(z)$ неприводим над полем $F_{2}$, $\theta^{*}(z)=\theta(z)$

(2) функциия $f$, рассматриваемая как $\left(\left(\varphi * \theta^{t-1}\right), \theta, L\right)$-композиияи, удовлетворяет одному из условий 1-6 теоремы 8;

(3) $(\lambda(z), z+1)=1$, либо $L(e)=0$. 
Тогда справедливы следующие утверждения.

(1) Все функции множества $M(f)$ являются попарно эквивалентными.

(2) $|M(f)|=\sum_{g \in M_{\lambda}(f)}|Z(g)|$.

(3) Если функция $f(x)$ отлична от функциии $f(x+e)+1$, то

$$
|M(f)|=|Z(f)|(t+1) .
$$

Доказательство. Эквивалентность функций множества $M(f)$ вытекает из теоремы 9 и леммы 10.

Пусть $g, h \in M_{\lambda}(f)$,

$$
\begin{aligned}
& g(x)=\left(\theta^{p} *\left(\varphi * \theta^{t-p}\right)\right)(x)+L(x), \\
& h(x)=\left(\left(\theta^{q} *\left(\varphi * \theta^{t-q}\right)\right)(x)+L(x), \quad p \neq q .\right.
\end{aligned}
$$

Покажем, что

$$
Z(g) \cap Z(h)=\varnothing .
$$

Действительно, положим для определенности, что $p>q$. Тогда, с учетом леммы 3 , функции $g$ и $h$ можно представить в виде

$$
\begin{aligned}
& h(x)=\left(\left(\Theta^{q} *\left(\varphi * \theta^{t-q}\right)\right)(x)+L(x)=\left(\left(\theta^{q} *\left(\varphi * \theta^{t-p}\right)\right) * \theta^{p-q}\right)(x)+L(x),\right. \\
& g(x)=\left(\left(\theta^{p} *\left(\varphi * \theta^{t-p}\right)\right)(x)+L(x)=\left(\theta^{p-q} *\left(\theta^{q} *\left(\varphi * \theta^{t-p}\right)\right)\right)(x)+L(x) .\right.
\end{aligned}
$$

Следовательно, функции $h$ и $g$ можно рассматривать как $\left(\theta^{q} *\left(\varphi * \theta^{t-p}\right), \theta^{p-q}, L\right)-$ и $\left(\theta^{p-q}, \theta^{q} *\left(\varphi * \theta^{t-p}\right), L\right)$-композицию соответственно. С учетом теоремы 10 получаем, что $Z(g) \cap Z(h)=\varnothing$. Следовательно,

$$
|M(f)|=\left|\bigcup_{g \in M_{\lambda}(f)} Z(g)\right|=\sum_{g \in M_{\lambda}(f)}|Z(g)| .
$$

Пусть теперь функция $f(x)$ отлична от функции $f(x+e)+1$. Покажем, что в этом случае при любой функции $g \in M_{\lambda}(f)$ мощности множеств $Z(g)$ и $Z(f)$ совпадают. По лемме 11 , функция $g(x)$ будет отлична от функции $g(x+e)+1$. Пусть

$$
g(x)=\left(\left(\theta^{p} *\left(\varphi * \theta^{t-p}\right)\right)(x)+L(x), \quad 0 \leqslant p \leqslant t .\right.
$$

Так как

$$
\left(\theta^{p}\right)^{*}(z)=\left(\theta^{*}(z)\right)^{p}=\theta^{p}(z),
$$

по лемме 12 функция $g(x)$ будет отлична от функции $g_{\nabla}(x)$ в том и только в том случае, если функция

$$
f(x)=\left(\varphi * \theta^{t}\right)(x)+L(x)=\left(\left(\varphi * \theta^{t-p}\right) * \theta^{p}\right)(x)+L(x)
$$

будет отлична от $f_{\nabla}(x)$. Аналогичным образом (с учетом леммы 13 ) показывается, что функция $g(x)$ будет отлична от функции $g_{\nabla}(x+e)+1$ в том и только том случае, если функция $f(x)$ будет отлична от функции $f_{\nabla}(x+e)+1$. Следовательно,

$$
|Z(g)|=|Z(f)|, \quad|M(f)|=|Z(f)|(t+1) .
$$

На этом теорема полностью доказана. 


\section{6. Заключительные замечания}

Отметим, что с точки зрения конкретного расчета цикловой структуры соответствующих нелинейных регистров сдвига особый интерес представляют классы эквивалентности, в которых наряду с нелинейными имеются и линейные функции обратной связи. Например, все автономные двоичные регистры сдвига длины $n$, обладающие циклом длины $2^{n}-1$, являются изоморфными, и среди них имеются линейные (с помощью которых вырабатываются линейные рекуррентные последовательности максимального периода). Однако, использующийся в работе способ построения эквивалентных функций на основе $(\varphi, \lambda, L)$ - и $(\lambda, \varphi, L)$-композиций приводит к классам эквивалентных функций $M(f)$, которые имеют одну и ту же степень нелинейности. Таким образом, в случае нелинейной функции $f$ в построенных классах эквивалентности $M(f)$ нет линейных функций. Это приводит к необходимости использования вычислительной техники для определения конкретной цикловой структуры регистров сдвига, соответствующих заданному классу эквивалентности (в виду отсутствия к настоящему моменту эффективных теоретических методов расчета).

Пусть, например,

$$
\lambda(z)=\sum_{i=1}^{m} a_{i} z^{i-1}=(1+z)^{n-3} \in F_{2}[z], \quad m=n-2,
$$

и функция $f(x)=f\left(x_{1}, x_{2}, \ldots, x_{n}\right)$ имеет вид

$$
f(x)=\left(\sum_{i=1}^{m} a_{i} x_{i+1}\right)\left(\sum_{i=1}^{m} a_{i} x_{i+2}\right)+L(x),
$$

где функция

$$
L=L\left(x_{1}, x_{2}, \ldots, x_{n}\right)=x_{1}+L_{*}\left(x_{2}, x_{3}, \ldots, x_{n}\right)
$$

является линейной, зависящей существенным образом от переменной $x_{1}$ и принимающей нулевое значение на векторе $e=(1,1, \ldots, 1)$. Тогда мощность соответствующего класса эквивалентных функций $M(f)$ равна $4(n-2)$ или $2(n-2)$ в зависимости от того, различны функции $L_{*}\left(x_{2}, x_{3}, \ldots, x_{n}\right)$ и $L_{*}\left(x_{n}, x_{n-1}, \ldots, x_{2}\right)$ или они совпадают. При этом число функций $f$ вида (25), отвечающих различным классам $M(f)$, равно $2^{n-3}$ при нечетном $n$ и равно $2^{n-3}+2^{(n-4) / 2}$ при четном $n$.

В соответствии с результатами предыдущего раздела элементы множества $M(f)$ задаются как объединение множеств $Z\left(g_{\Theta, \nu}\right)$ по всем функциям $g_{\Theta, v}:\left(F_{2}\right)^{n} \rightarrow F_{2}$ вида

$$
g_{\theta, v}(x)=(\theta *(\varphi * v))(x)+L(x),
$$

где многочлены $\theta$ и $v$ удовлетворяют равенству

$$
\theta(z) v(z)=(1+z)^{n-3} .
$$

Пусть

$$
\begin{array}{llrl}
\theta(z) & =\sum_{i=1}^{m} \alpha_{i} z^{i-1}, & \alpha_{1}=\alpha_{m}=1, \\
v(z) & =\sum_{i=1}^{n-m-1} \beta_{i} z^{i-1}, & \beta_{1}=\beta_{n-m-1}=1 .
\end{array}
$$


Тогда из (26) получаем, что

$$
g_{\theta, v}(x)=\sum_{i=1}^{m} \alpha_{i}\left[\left(\sum_{j=1}^{n-m-1} \beta_{j} x_{i+j}\right)\left(\sum_{j=1}^{n-m-1} \beta_{j} x_{i+j+1}\right)\right]+L(x) .
$$

С помощью расчетов на ЭВМ для $n \leqslant 24$ были найдены все функции $f$ вида (25), являющиеся представителями различных классов эквивалентности $M(f)$ и отвечающие регистрам сдвига с циклом длины $2^{n}-1$, и для $25 \leqslant n \leqslant 27$ найдены примеры таких функций. Данные результаты приведены в приложении.

В заключение рассмотрим одну более общую конструкцию, которая, возможно, позволит строить эквивалентные функции с разной степенью нелинейности.

Теорема 12. Пусть $\varphi:\left(F_{2}\right)^{n-m+1} \rightarrow F_{2}, \psi:\left(F_{2}\right)^{m-1} \rightarrow F_{2}-$ произвольные булевы функичии от соответствующего числа аргументов,

$$
\begin{gathered}
f\left(x_{1}, x_{2}, \ldots, x_{n}\right)=x_{1}+\varphi\left(\psi\left(x_{1}, x_{2}, \ldots, x_{m-1}\right)+x_{m},\right. \\
\left.\ldots, \psi\left(x_{n-m+1}, \ldots, x_{n-1}\right)+x_{n}\right) \\
+\psi\left(x_{n-m+2}, \ldots, x_{n}\right), \\
g\left(x_{1}, x_{2}, \ldots, x_{n}\right)=x_{1}+\psi\left(\varphi\left(x_{1}, x_{2}, \ldots, x_{n-m+1}\right)+x_{n-m+2},\right. \\
\left.\ldots, \varphi\left(x_{m-1}, \ldots, x_{n-1}\right)+x_{n}\right) \\
+\varphi\left(x_{m}, x_{m+1}, \ldots, x_{n}\right), \\
A=\left(A_{1}, A_{2}, \ldots, A_{n}\right):\left(F_{2}\right)^{n} \rightarrow\left(F_{2}\right)^{n}, \\
A_{1}=\psi\left(x_{1}, x_{2}, \ldots, x_{m-1}\right)+x_{m}, \\
A_{i+1}(x)=A_{i}\left(\delta_{t}(x)\right), \quad i=1,2, \ldots, n-1 .
\end{gathered}
$$

Тогда отображение А биективно, и выполняется равенство $A \delta A^{-1}=\delta_{g}$.

Доказательство. Расписав подробнее координатное представление отображения $A$, получаем, что

$$
\begin{aligned}
A_{1} & =\psi\left(x_{1}, x_{2}, \ldots, x_{m-1}\right)+x_{m}, \\
A_{1} & =\psi\left(x_{2}, x_{3}, \ldots, x_{m}\right)+x_{m+1}, \\
& \ldots \\
A_{n-m+1} & =\psi\left(x_{n-m+1}, \ldots, x_{n-1}\right)+x_{n}, \\
A_{n-m+2} & =x_{1}+\varphi\left(A_{1}, A_{2}, \ldots, A_{n-m+1}\right), \\
A_{n-m+3} & =x_{2}+\varphi\left(A_{2}, A_{3}, \ldots, A_{n-m+2}\right), \\
& \ldots \\
A_{n} & =x_{m-1}+\varphi\left(A_{m-1}, A_{m}, \ldots, A_{n-1}\right) .
\end{aligned}
$$

Из последних $m-1$ уравнений системы (27) находятся переменные $x_{1}, x_{2}, \ldots, x_{m-1}$. После чего оставшиеся переменные последовательно находятся из первых $n-m+1$ уравнений. Тем самым, отображение $A$ биективно. С учетом леммы 1 для доказательства теоремы теперь достаточно убедиться в справедливости равенства

$$
g(A(x))=A_{n}\left(\delta_{f}(x)\right)
$$


Справедливо равенство

$$
\begin{aligned}
g(A(x))=A_{1}+\psi\left(\varphi\left(A_{1}, A_{2}, \ldots, A_{n-m+1}\right)+A_{n-m+2},\right. & \\
& \left.\ldots, \varphi\left(A_{m-1}, \ldots, A_{n-1}\right)+A_{n}\right)+\varphi\left(A_{m}, A_{m+1}, \ldots, A_{n}\right)
\end{aligned}
$$

С другой стороны, с учетом (27) получаем, что

$$
\begin{aligned}
A_{n}\left(\delta_{f}(x)\right)=x_{m} & +\varphi\left(A_{m}, A_{m+1}, \ldots, A_{n}\right) \\
=A_{1} & +\psi\left(\varphi\left(A_{1}, A_{2}, \ldots, A_{n-m+1}\right)\right. \\
& \left.+A_{n-m+2}, \ldots, \varphi\left(A_{m-1}, \ldots, A_{n-1}\right)+A_{n}\right) \\
& +\varphi\left(A_{m}, A_{m+1}, \ldots, A_{n}\right) .
\end{aligned}
$$

Следовательно, равенство (28) выполнено, и теорема доказана.

\section{7. Приложение}

Ниже приведен перечень классов $M(f)$ квадратичных функций обратной связи регистров сдвига, обладающих циклом длины $2^{n}-1$, где $n-$ длина регистра, $L-$ число, определяющее линейную часть функции обратной связи.

\begin{tabular}{r|l}
$n$ & \multicolumn{1}{|c}{$L$} \\
\hline 4 & 1 \\
5 & $1,2,11$ \\
7 & $1,37,55$ \\
8 & $13,47,59$ \\
9 & $26,44,133$ \\
10 & 87,351 \\
11 & 8 \\
12 & $481,575,577,995$ \\
13 & $949,1822,1838,2225$ \\
14 & 206,5827 \\
15 & $6453,9059,12839$ \\
16 & 21237 \\
17 & 12269 \\
18 & $18662,31118,68519,93839$ \\
19 & $20796,34476,84057,174551$ \\
20 & 82642 \\
21 & 849314 \\
22 & 765840 \\
23 & 1788112 \\
24 & $2946962,3375584,6852738,6901516,7970725,8065036$ \\
25 & 6529570 \\
26 & 5105416 \\
27 & 11750144 \\
&
\end{tabular}

Замечание 4. Для $n \leqslant 24$ в приложении приведены результаты полного перебора всех возможных вариантов для $L$.

Для $25 \leqslant n \leqslant 27$ эксперименты проводились до первого успеха, то есть до первого нахождения линейной части $L$ соответствующей функции обратной связи регистра сдвига, обладающего циклом длины $2^{n}-1$. 
При $n=6$ искомое множество оказалось пустым.

Замечание 5. Пусть двоичный вектор $b_{1}, b_{2}, \ldots, b_{n-1}$ отвечает двоичной записи числа $L$, то есть

$$
L=b_{1}+b_{2} 2+b_{3} 2^{2}+\ldots+b_{n-1} 2^{n-2} .
$$

Тогда линейная булева функция $L_{*}=L_{*}\left(x_{1}, x_{2}, \ldots, x\right)$, соответствующая числу $L$, имеет вид

$$
L_{*}\left(x_{2}, x_{3}, \ldots, x_{n}\right)=b_{1} x_{2}+b_{2} x_{3}+\ldots, b_{n-1} x_{n} .
$$

Функция $f(x)=f\left(x_{1}, x_{2}, \ldots, x_{n}\right)$, отвечающая классу эквивалентности $M(f)$, имеет вид

$$
f(x)=\left(\sum_{i=1}^{m} a_{i} x_{i+1}\right)\left(\sum_{i=1}^{m} a_{i} x_{i+2}\right)+b_{1} x_{2}+b_{2} x_{3}+\ldots+b_{n-1} x_{n}+x_{1},
$$

где

$$
\lambda(z)=\sum_{i=1}^{m} a_{i} z-i-1=(1+z)^{n-3} \in F_{2}[z], \quad m=n-2 .
$$

При этом мощность соответствующего класса эквивалентных функций оказалась равной $4(n-2)$, так как для найденных чисел $L$ соответствующие функции $L_{*}\left(x_{2}, x_{3}, \ldots, x_{n}\right)$ и $L_{*}\left(x_{n}, x_{n-1}, \ldots, x_{2}\right)$ всегда различны.

\section{Список литературы}

1. Гантмахер Ф. Р., Теория матриц. Наука, Москва, 1988.

2. Колмогоров А. Н., Фомин С. В., Элементы теории функций и функционального анализа. Наука, Москва, 1972.

3. Холл М., Комбинаторика. Мир, Москва, 1970.

4. Berlekamp E. R., Distribution of cyclic matrices in finite field. Duke Math. J. (1966) 33, 45-48.

5. Dickson L. E., Linear groups with an exposition of the Galois field theory. Teubner, Leipzig, 1901.

6. Fredricksen H., A class of nonlinear de Bruijn cycles. J. Comb. Theory (1975) A19, 192-199.

7. Golomb S. W., Shift Register Sequences. Aegean Park, Laguna Hills, California, 1982.

8. Games R. A., Chan A. H., A fast algorithm for determining the complexity of a binary sequence with period $2^{n}$. IEEE Trans. Inf. Theory (1983) 29, 144-146.

9. Games R. A., There are no de Bruijn sequences of span $n$ with complexity $2^{n-1}+n+1$. J. Comb. Theory (1983) A34, 248-251.

10. Kjeldsen K., On the cycle structure of a set of nonlinear shift registers with symmetric feedback functions. J. Comb. Theory (1976) A20, 154-169.

11. Lempel A., On a homomorphism of the de Bruijn graph and its application to the design of feedback shift registers. IEEE Trans. Comput. (1970) 19, 1204-1209.

12. Lempel A., Etzion T., On the distribution of de Bruijn sequences of given complexity. IEEE Trans. Inf. Theory (1984) 30, 611-614.

13. Lempel A., Etzion T., Construction of de Bruijn sequences of minimal complexity. IEEE Trans. Inf. Theory (1984) 30, 705-709.

14. Zurawiecki J., Boolean shift-registers. Demonstr. Math. (1977) 10, 405-415.

15. Солодовников В. И., О полугруппе, порожденной автоматными отображениями обратимого автомата. Труды по дискретной математике (2001) 4, 231-242.

16. Глухов М. М., Елизаров В. П., Нечаев А. А., Алгебра. Гелиос АРВ, Москва, 2003.

Статья поступила 21.01.2008. 